\title{
Thermal/Structural Analysis and Frequency Shift Studies for the Spallation Neutron Source (SNS) Drift Tube Linac
}

\author{
L. Parietti ${ }^{\#}$, N. K. Bultman, and Z. Chen, LANL, Los Alamos, NM
}

\begin{abstract}
The drift tube linac of the Spallation Neutron Source accelerates the beam from $2.5 \mathrm{MeV}$ to $20 \mathrm{MeV}$ at the operating frequency of $402.5 \mathrm{MHz}$. This pulsed operating linac is about 9 meters long and consists of 84 cells with 83 permanent magnet quadrupole drift tubes. The DTL relies on the cooling system to zero out the frequency shift caused by RF heating on the cavity walls. Thermal expansion and frequency shift studies for three individual cells at a $3 \times 10^{6} \mathrm{~V} / \mathrm{m}$ accelerating gradient are performed via numerical simulations to help guide the design of the cooling channels.
\end{abstract}

\section{INTRODUCTION}

The Spallation Neutron Source (SNS) is an acceleratorbased facility that will be built at Oak Ridge National Laboratory by 2005 . The SNS will produce pulsed beams of neutrons by bombarding a mercury target with intense beams of $1-\mathrm{Gev}$ protons. This facility is being designed to meet the needs of the neutron scattering community in the United States.

The SNS project is a collaboration of five national laboratories. A full-energy linac injecting into an accumulator ring has been selected among several technology options for the accelerator system. The beam generated by an ion source is accelerated in the linac, accumulated and bunched in the ring structure, then extracted and transported to the target.

Los Alamos National Laboratory is responsible for the linac systems. The linac takes the beam from the frontend system and accelerates it from $2.5 \mathrm{MeV}$ to $1.0 \mathrm{GeV}$. It consists of a Drift-Tube Linac (DTL) that accelerates the beam to $20 \mathrm{MeV}$, a Coupled-Cavity Drift Tube Linac (CCDTL) that accelerates the beam to $87 \mathrm{MeV}$, and a Coupled-Cavity Linac (CCL) that accelerates the beam to $1 \mathrm{GeV}$. The DTL operates at a Radio Frequency (RF) of $402.5 \mathrm{MHz}$, while the CCDTL and CCL operate at 805 MHz.

Under normal operation (beam on), about $80 \%$ of the RF power is dissipated in the cavity walls. This amounts to $76 \mathrm{~kW}$ at a $7 \% \mathrm{RF}$ duty factor. The power dissipated causes thermal distortions (i. e., shape change) which result in a frequency shift. To maintain the desired resonance frequency, each cavity is cooled by forced water circulation.

This paper summarizes the frequency shift studies conducted for the SNS DTL. This DTL consists of one tank and 83 drift tubes that are cooled on two separate circuits. The thermal deformations resulting from RF heating are evaluated separately for both the tank and three individual drift tubes using finite element models. The frequency shift of these three cells are then computed based on the calculated deformations. Size and location of the cooling channels are designed to provide adequate cooling and resonant frequency control.

\section{SNS DRIFT TUBE LINAC DESCRIPTION}

The SNS DTL, shown in Fig. 1, is $8.3 \mathrm{~m}$ long and accelerates the beam from $2.5 \mathrm{MeV}$ to $20 \mathrm{MeV}$ at a $3 \times 10^{6}$ $\mathrm{V} / \mathrm{m}$ accelerating gradient. It consists of one tank, 84 cells and 83 drift tubes.

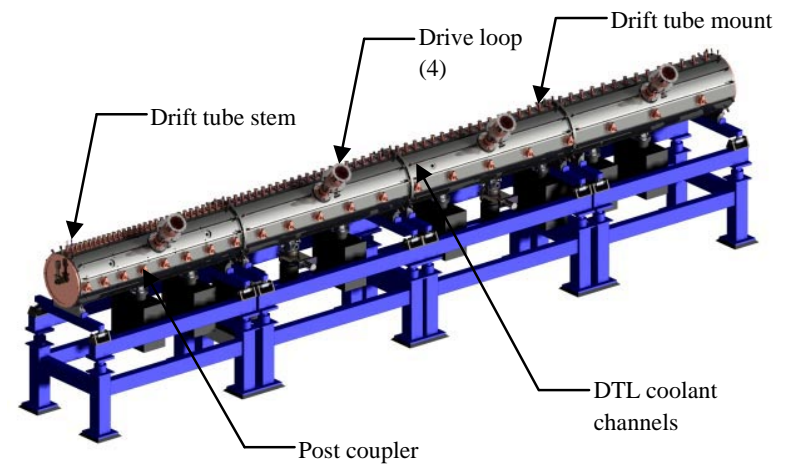

Figure 1: SNS Drift Tube Linac

The DTL tank combines vacuum envelope and RF structure, and provides mechanical rigidity to the assembly. The tank is made of 4 seamless carbon-steel cylinders with a finished wall thickness of about 2.8 inches. The inner diameters are machined and copperplated to achieve the final dimension and the required surface conductivity. The cylinders are about 2 meters long each and are bolted together with RF and vacuum seals at each joint.

Each of the 83 drift tubes contains a permanent magnet quadrupole. The geometry of the drift tubes (fig. 2) changes with their position along the DTL (since the energy level increases); the face angle increases from $3^{\circ}$ to $55^{\circ}$, while the length increases from $4.3 \mathrm{~cm}$ at $2.5 \mathrm{MeV}$ to $12.4 \mathrm{~cm}$ at $20 \mathrm{MeV}$. Figure 3 shows a cross-section of the highest energy drift tube. The drift tube body, made of copper, consists of 5 electron-beam (EB) welded parts. The body is brazed to the stainless steel stem before the magnet is inserted. The stem consists of two concentric 
tubes serving as inlet and outlet ducts for the cooling water.

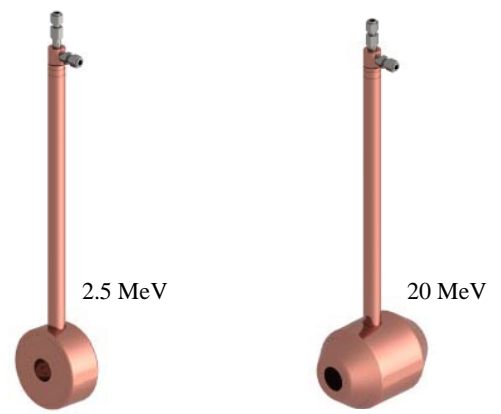

Figure 2: SNS DTL drift tubes at 2.5 and $20 \mathrm{MeV}$

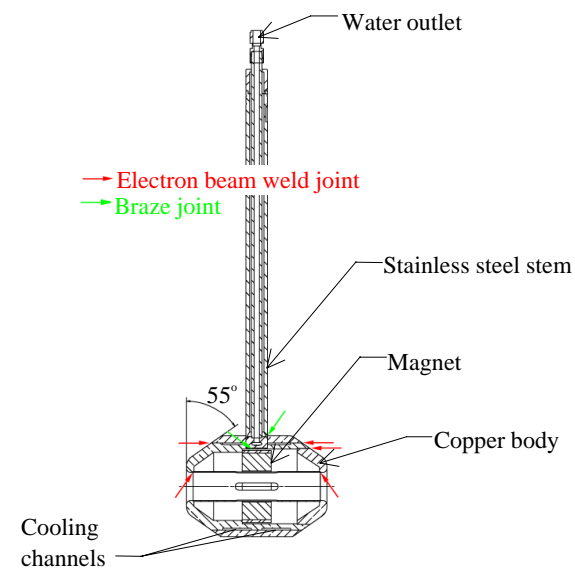

Figure 3: SNS DTL drift tube cross-section at $20 \mathrm{MeV}$

\section{TEMPERATURE DISTRIBUTIONS DUE TO RF HEATING}

\subsection{Tank Wall}

When the RF is turned on, about $32 \mathrm{~kW}$ are dissipated into the tank walls. To compensate for the thermal distortions caused by RF heating, the tank is cooled via 12 rectangular stainless-steel cooling channels ( 1 in wide by 0.5 inches high) that are bonded and clamped in machined groves on the tank walls. The cooling channels are located 1 inch away from the inside wall. An average $30^{\circ}$ angle separates two cooling channels. Taking advantage of the problem periodicity, a two-dimensional finite element model of a $15^{\circ}$ tank section was created using COSMOS/M. The water velocity is assumed to be 0.5 $\mathrm{m} / \mathrm{s}$, which gives a heat transfer coefficient of 2511 $\mathrm{W} / \mathrm{m}^{2} \mathrm{~K}$ for this channel geometry. This heat transfer coefficient is applied on the outer edges of the cooling channels to simulate a forced convection boundary condition. All the other outer surfaces are assumed adiabatic.

Figure 4 shows the tank temperature distribution when the water running through the cooling channels is at $293.2 \mathrm{~K}$. The average tank temperature is $297.4 \mathrm{~K}$, while the average temperature on the inside wall is $298.5 \mathrm{~K}$. These calculated values are used later to evaluate the frequency shift of a DTL cell in the drift tube model (Section 4).

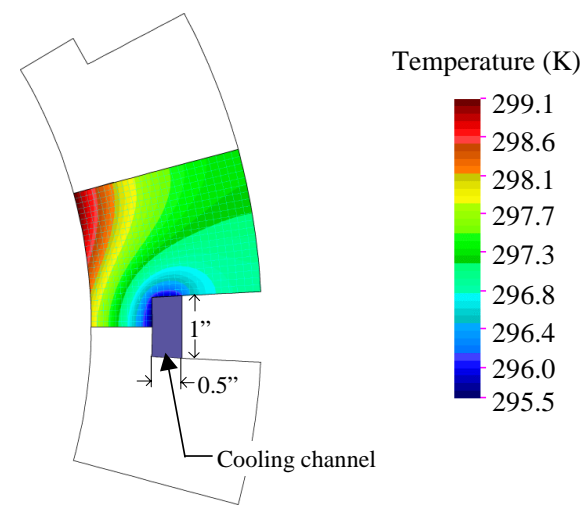

Figure 4: Tank temperature distribution

\subsection{Drift Tube}

Cooling channels are machined in the drift tube body. The flow of water is fed through the outer tube of the stem, splits in half, circulates around the drift tube body and exits trough the stem inner tube.

Two-dimensional axisymmetric models for three individual drift tubes at 2.5, 10.5 and $20 \mathrm{MeV}$ were built using COSMOS/M. The amount of heat dissipated on the drift tube outside walls is not uniform. The heat fluxes resulting from RF heating are given by the physics code SUPERFISH.

Figure 5 shows the temperature distribution for the high energy drift tube assuming a water temperature of $293.2 \mathrm{~K}$.

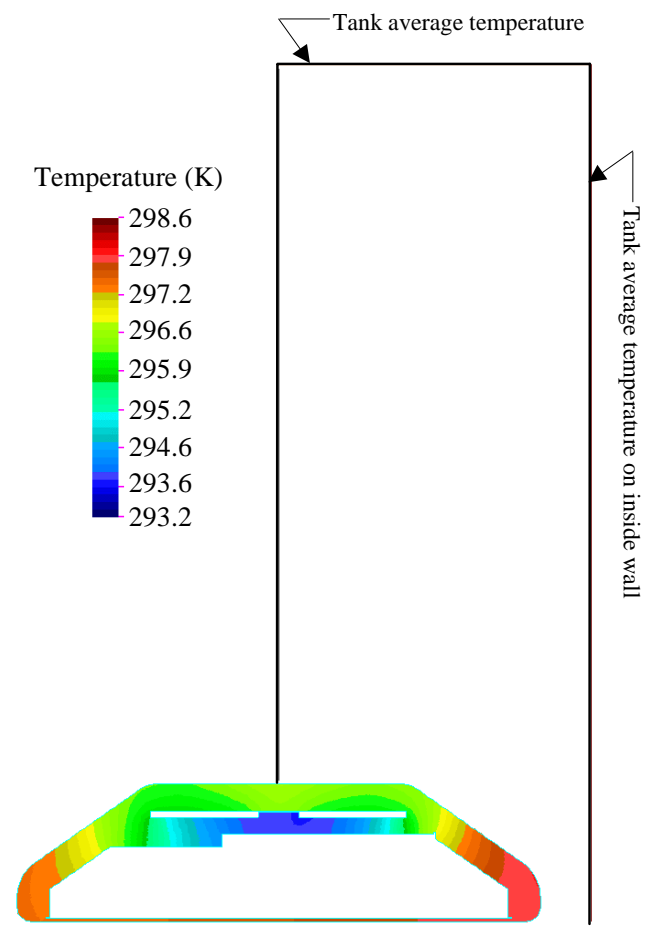

Figure 5: High energy drift tube temperature distribution 
The cooling channels are 1 inch wide by 0.06 inch high. They are placed as close as possible from the outer edges to provide maximum cooling. At the interface between parts, the only heat transfer paths are across the EB welds. Small physical gaps are conservatively included in the model when there is only surface contact between two parts. The flow rate through the drift tube stem is limited to $2.19 \mathrm{gpm}$ to avoid having an impingement velocity on the drift tube body greater than $2.5 \mathrm{~m} / \mathrm{s}$ which would cause material erosion. This flow rate corresponds to a water velocity of $1.7 \mathrm{~m} / \mathrm{s}$ and gives a heat transfer coefficient of $9607 \mathrm{~W} / \mathrm{m}^{2} \mathrm{~K}$.

\section{FREQUENCY SHIFT STUDIES}

Thermal distortions caused by RF heating result in a frequency shift as the shape of the cavity changes. For any given cell, the change in frequency shift can be calculated using the Slater perturbation theory. The change in frequency $d f$ is a function of the volume change of an infinitesimal volume $d V$ laying on the RF surface, as well as the electromagnetic field $H$ and the electric field $E$ for this volume. The frequency shift is given by:

$$
\frac{d f}{f}=\frac{\int\left(\mu H^{2}-\varepsilon E^{2}\right) d V}{4 U},
$$

where $\mu$ and $\varepsilon$ are the free space permeability and permittivity, respectively and $U$ is the cavity stored energy.

For a DTL, both the tank deformations (radial and longitudinal) and the drift tube deformations contribute to the frequency shift. To account for the tank deformations, beam elements are added to the drift tube model described in section 3.2. These beam elements, as shown in Fig. 5, are set to a uniform temperature corresponding to the inside tank wall temperature and the average tank wall temperature to simulate the radial and the longitudinal growth of the tank. The thermal deformations corresponding to the drift tube and the tank temperature profile are then solved using the COSMOS/M finite element package. A separate Fortran code reads the displacements output by the finite element package and computes the frequency shift of the cell. The frequency shift for the high energy drift tube for a flow rate of 2.19 gpm is $-31 \mathrm{KHz}$.

The cooling system can be used in several ways to zero out the frequency shift caused by RF heating. The resonant frequency can be controlled by either changing the temperature of the water in the tank circuit or by changing the water temperature in the drift tube circuit. The frequency shift studies can help in the decision making. The sensitivity of the drift tube circuit to water temperature changes was investigated using the model described above. The drift tube circuit frequency shift is 5 to 8 times more sensitive to water temperature changes than the tank circuit ( 5 times for the low energy drift tube and 8 times for the high energy one). Furthermore, compensating for frequency shift by regulating the tank cooling water would require operating the tank walls at exceedingly high temperatures (300 to $373 \mathrm{~K}$ ). Varying the water in the tank circuit to zero out the cavity frequency shift is therefore not practical. Instead, the cooling water temperature in the drift tube circuit will be dynamically controlled to maintain resonance.

However, the heat dissipated on the drift tube outside walls increases as the energy level increases $(0.2 \mathrm{~kW}$ for the low energy drift tube compared to $0.5 \mathrm{~kW}$ for the high energy drift tube). To avoid field errors and frequency mismatch, the frequency shift needs to be the same for all the DTL cells. This will be achieved by adjusting the flow rate using an orifice plate for each individual drift tube. Figure 6 shows the frequency shift for the cells containing drift tubes \#1, \#53 and \#83 as a function of the flow rate. In addition to balancing the flow rate between the 83 drift tubes, the width of the cooling channels is tailored to keep the flow turbulent.

From a practical standpoint, the structure will be tuned during final assembly to $+30 \mathrm{KHz}$ to give a nominal 402.5 $\mathrm{MHz}$ resonance during powered operation. The temperature of the flow balanced system can also be adjusted during operation to correct for additional frequency shift. Its sensitivity is about $-7.5 \mathrm{KHz} / \mathrm{K}$.

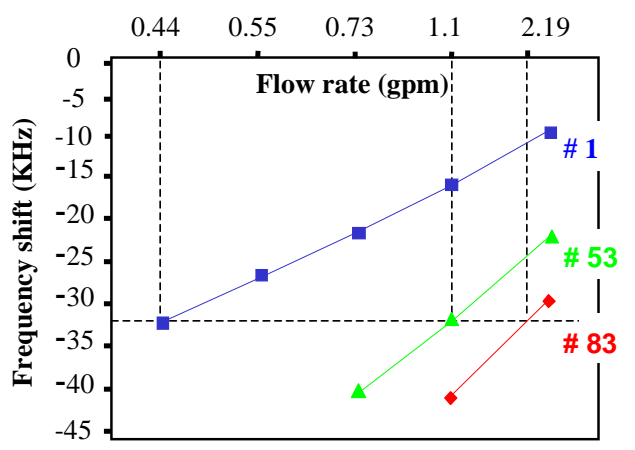

Figure 6: Flow balancing between drift tubes to maintain a uniform frequency shift across the DTL cells

\section{CONCLUDING REMARKS}

The DTL resonant frequency must be regulated by the cooling system. Frequency shift studies for three DTL cells at 2.5, 10.5 and $20 \mathrm{MeV}$ were performed to help design the cooling channels. Resonance will be maintained by dynamically adjusting the water temperature in the drift tube circuit. A uniform frequency shift for all 84 cells can be obtained by balancing the flow rate and tailoring the cooling channels for each individual drift tube.

This work is supported by the Department of Energy and the Oak Ridge National Laboratory.

\# E-mail: parietti@lanl.gov 